\title{
A family cluster of nitrite poisoning, Suzhou City, Jiangsu Province, China, 2013
}

\author{
Ruiping Wang, ab Chen-gang Teng, ${ }^{c}$ Ning Zhang, ${ }^{c}$ Jun Zhang ${ }^{c}$ and George Conway ${ }^{d}$ \\ Correspondence to Chen-gang Teng (e-mail: sztcg123456@yahoo.com.cn) and Rui-ping Wang (e-mail: w19830901@126.com).
}

Background: In April 2013, a hospital in Suzhou City notified authorities of a patient with nitrite poisoning with two other family members who had similar toxic symptoms five days prior. We investigated the event to identify the cause, source and possible route of contamination.

Methods: A case was defined as any person living in the Yang Shan Hua Yuan community who had been diagnosed with cyanoderma and food poisoning symptoms from 15 to 25 April 2013. Active case finding was conducted by interviewing community residents and reviewing medical records from local clinics; information was then retrospectively collected on the patient's food history, cooking procedures and food sources.

Results: We identified three nitrite poisoning cases, one male and two females, from the same family. The time between dinner and onset of illness was less than an a hour. A retrospective survey showed that a substance presumed to be sugar mixed with asparagus on 17 April and with stir-fried asparagus on 21 April wasthe suspected contaminant. The presumed sugar came from a clean-up of a neighbouring rental house. Nitrite was detected in a vomitus sample, the sugar substance and two leftover food samples.

Conclusion: This family cluster of nitrite poisoning resulted from the mistaken use of nitrite as sugar to cook dishes. We recommend that sodium nitrite be dyed a bright colour to prevent such a mistake and that health departments strengthen food hygiene education to alert people about the danger of eating unidentified food from an unknown source.

$\mathrm{N}$ itrite is the general term of a category of inorganic compounds, mainly sodium nitrite. This white to slight yellowish crystalline powder is very soluble in water, hygroscopic and has been widely used in industry and construction. ${ }^{1}$ Since the early 1900s, sodium nitrite has been used to inhibit growth of diseasecausing microorganisms, give taste and colour to meat and inhibit lipid oxidation that leads to rancidity. ${ }^{2}$

Sodium nitrite can be toxic in high amounts for humans; ${ }^{3}$ acute nitrite intoxication can occur after ingestion of $200 \mathrm{mg}$ to $500 \mathrm{mg}$ with an incubation period commonly within one hour, ranging from 20 minutes to three hours. Symptoms include dizziness; fatigue; tightchest; nausea; vomiting; cyanosis in the lips, fingernails and skin; tachycardia; unconsciousness; coma; and even death. ${ }^{4,5}$ Nitrite can cause methemoglobinemia, which makes red blood cells lose their oxygen-carrying ability, reducing the amount of oxygen that is released from haemoglobin. In China, acute nitrite poisoning is commonly caused by mistaking nitrite for salt or from eating large amounts of vegetables or meat with a high nitrite content. ${ }^{6-9}$

In April 2013, a hospital in Suzhou City notified Suzhou Center for Disease Control and Prevention of a patient in a coma from nitrite poisoning with two family members who had similar toxic symptoms five days prior. We conducted an investigation to identify the cause of the nitrite poisoning, to identify he source of the potential toxin and possible contamination routes and to recommend control measures to prevent similar events in the future.

\section{METHODS}

A case was defined as any person residing in the Yang Shan Hua Yuan (YSHY) community with cyanoderma (lip, tongue tip, fingertip, conjunctiva, face or the whole body) and with at least one of the following symptoms: dizziness, headache, fatigue, tachycardia, drowsiness, nausea, vomiting, abdominal pain or diarrhoea from

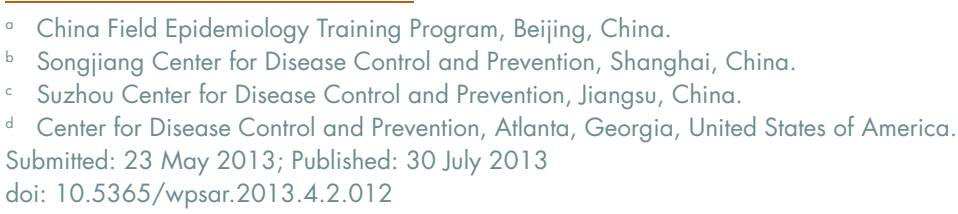


Figure 1. Timeline of dinner and duration of illness for a family cluster of nitrate poisoning, Suzhou District, Jiangsu, China, 2013

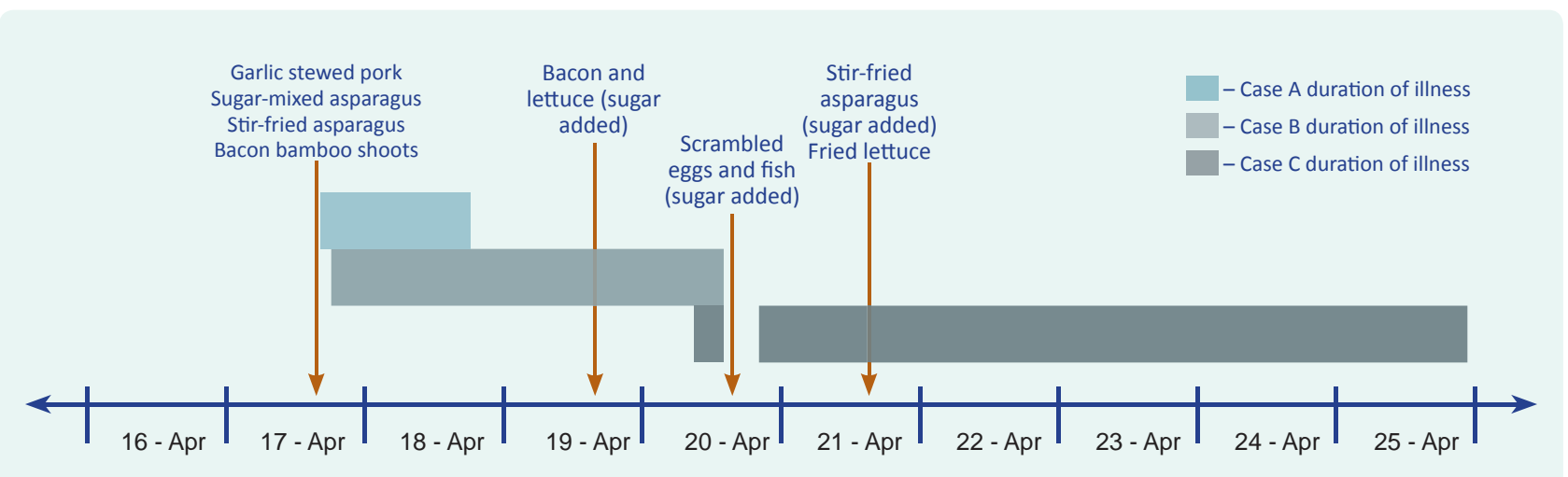

15 to 25 April 2013. Active case finding was conducted by interviewing residents in the YSHY community and reviewing the medical records from all local clinics.

We conducted in-depth interviews with the patients and their families using a structured questionnaire regarding food and water consumption and other environmental factors. The question focused on meals eaten 12 hours before patients' clinical onset, specific cooking procedures of suspected foods and sources of food and condiments. The Ethics Committee of the Suzhou Center for Disease Control and Prevention approved this investigation.

One venous blood sample and one vomitus sample were collected from the patients; we also collected $10 \mathrm{~g}$ salt, $20 \mathrm{~g}$ chicken powder, $15 \mathrm{~g}$ of sugar substance, $20 \mathrm{~g}$ monosodium glutamate, $100 \mathrm{~g}$ uncooked asparagus, $50 \mathrm{~g}$ bacon, $150 \mathrm{~g}$ leftover stir-fried asparagus and $100 \mathrm{~g}$ leftover fried lettuce from a dinner before illness onset. Samples were tested for nitrite content or density using the Griess-Saltzman method according to the Chinese national standard (GB/T5009.33-2010). ${ }^{10}$

\section{RESULTS}

Three cases were identified: one male and two females from the same family. Clinical features included lip cyanosis (3/3), dizziness (3/3), tachycardia (3/3), nausea $(3 / 3)$, vomiting $(1 / 3)$, unconsciousness $(1 / 3)$ and coma $(1 / 3)$.

Case $A$ and Case $B$ ate dinner together on 17 April. Case A, a 43-year-old male, experienced symptoms about 50 minutes after dinner, including lip cyanosis, dizziness, cardiopalmus and nausea. He went to a local hospital for treatment and was transferred to the emergency department of a Suzhou city hospital where he was intravenously injected with methylene blue (MB). He recovered and was discharged the next day. Case $B$, the 21-year-old daughter of Case $A$, became ill about an hour after dinner; she felt dizzy, nauseated and had an accelerated heartbeat. She went to the same local hospital for treatment, was transferred to the emergency department of a Suzhou city hospital and received MB treatment by intravenous injection. She was hospitalized for three days (Figure 1).

Case C, a 68-year-old female, is the mother of Case A. On 20 April, she was visiting her granddaughter (Case B) and was asked to see a doctor due to her lip cyanosis. She was given a vitamin $C$ intravenous drip treatment, felt better and returned home. On 21 April, she ate dinner with her family and about 40 minutes later felt dizzy, nauseated and became unconscious. She was sent to the local hospital for treatment, fell into a coma, and was admitted to a Suzhou city hospital intensive care unit for four days (Figure 1).

As nitrite poisoning was diagnosed, case interviews focused on the meals eaten and activities of the three cases and their family members for 17 April and 21 April (Figure 1). On 17 April, the family dinner was composed of four dishes (garlic stewed pork, sugar mixed asparagus, stir-fried asparagus and bacon bamboo shoots). Cases A and B ate all four dishes; Case $C$ and a non-ill family member ate three dishes but not the sugar mixed asparagus. Thus the sugar mixed asparagus was considered the probable contaminated food since Case $A$ and $B$ were ill after this meal. 
Table 1. Nitrite density of samples collected in a family cluster of nitrite poisoning, Suzhou City, Jiangsu, China, 2013

\begin{tabular}{lr}
\hline \multicolumn{1}{c}{ Sample } & Density(mg/kg) \\
\hline Patient & 173 \\
Vomitus & 0.30 \\
Venous blood & \\
Food & 714286 \\
Sugar substance & 9071 \\
Stir-fried asparagus (leftover) & 474 \\
Chicken powder & 187 \\
Uncooked asparagus & 14 \\
Fried lettuce (leftover) & 6.7 \\
Bacon & 2.2 \\
Salt & 1.9 \\
Monosodium glutamate & \\
\hline
\end{tabular}

On 21 April, Case A, Case C and the same non-ill family member had dinner together, eating stir-fried asparagus and fried lettuce. All three family members ate both dishes, but Case $A$ and the nonill family member mainly ate the fried lettuce while Case $\mathrm{C}$ mainly ate the stir-fried asparagus. She became ill later that night, making the stir-fried asparagus another probable contaminated food.

Interviews revealed that the non-ill family member assisted a neighbour in cleaning a rental house on 15 April; he found an unmarked plastic bag of what was presumed to be sugar and took it home as a condiment. Case $\mathrm{C}$ used this to cook the sugar mixed asparagus on 17 April and the stir-fried asparagus on 21 April but not for the other dishes. Case A reported that he also added a little of the presumed sugar when he cooked lettuce on 19 April and fish on 20 April.

Laboratory testing showed that the sugar substance was high-density nitrite $(714286 \mathrm{mg} / \mathrm{kg})$. The nitrite content of the leftover stir-fried asparagus from the 21 April dinner was $9071 \mathrm{mg} / \mathrm{kg}$ and the vomitus sample was $173 \mathrm{mg} / \mathrm{kg}$. The nitrite content of the leftover fried lettuce from the 21 April dinner was lower at $14 \mathrm{mg} / \mathrm{kg}$ (Table 1).

\section{DISCUSSION}

In this investigation, strong laboratory and epidemiological evidence led to the conclusion that the mistaken use of nitrite as sugar in food preparation was the source of this family cluster of nitrite poisoning. The presumed sugar was identified as high-density nitrite by laboratory testing, and the nitrite contents in the suspected food of stir-fried asparagus as well as patient vomitus were also high. It is unlikely that other food items were the cause of the nitrite poisoning as the three cases had no other common food exposures before onset.

It appears that Case C suffered minor nitrate poisoning before her hospitalization after the shared second meal on 21 April. Case A reported adding a little presumed sugar when he cooked lettuce on 19 April; Case $\mathrm{C}$ ate the leftover lettuce on 20 April, possibly explaining why she had lip cyanosis on 20 April.

The epidemiological evidence in this investigation was clear for the first incident because on 17 April Case A and Case B both ate the nitrite-contaminated sugar mixed asparagus; the other two family members present were not poisoned as neither ate the contaminated food. Similarly, for the second incident on 21 April, Case $\mathrm{C}$ mainly ate the nitrite-contaminated stir-fried asparagus and then became ill. However, the other two family members present at this meal were not poisoned even though they ate some contaminated food. A possible explanation for Case $A$ is that he mainly ate the fried lettuce at this meal, which was not contaminated by nitrite, plus he had received MB treatment four days previously which may have left some residue in his body making him asymptomatic. The nonill family member mainly ate the fried lettuce and also consumed some nitrite-contaminated food on 21 April. However, he was 71 years old with moderate Alzheimer's disease, perhaps making it less likely for him to report mild symptoms to his family.

The nitrite content in the chicken powder sample exceeded the national standard for condiments. The environmental investigation determined that the family shared one spoon for the sugar and the chicken powder, making it plausible that some nitrite may have gotten into the chicken powder. The nitrite content in the uncooked asparagus sample was also high because it was pre-treated with the presumed sugar on 21 April.

In recent years, nitrite intoxication has happened frequently in China despite risk communication efforts. This is probably because of the similar characteristics 
and appearance of sodium nitrite to salt and sugar; it tastes salty and is widely used as a food additive. In China, sodium nitrite can be easily purchased, and many residents are unaware of the potential harm of sodium nitrite. From this investigation we found weaknesses in the regulations, surveillance and supervision work. We recommend that sodium nitrite be dyed a bright colour, such as red, blue or yellow, to avoid mistaking it for plain salt or sugar. Health departments should carry out health education on food hygiene and food safety, especially in rural areas, to improve residents' knowledge and awareness. The Food and Drug Administration should strengthen the supervision of sodium nitrite sales, strictly manage and control sodium nitrite use for industry and as a food additive, and require the nitrite industries to add obvious warning labels on packages of nitrite.

\section{Conflicts of interest}

None declared.

\section{Funding}

None.

\section{References:}

1. Laue W et al. Nitrates and nitrites. Ullmann's Encyclopedia of Industrial Chemistry, 2002, 265-280.

2. Sindelar JJ, Milkowski AL. Human safety controversies surrounding nitrate and nitrite in the diet. Nitric Oxide, 2012, 26:259-266. doi:10.1016/j.niox.2012.03.011 pmid:22487433

3. Locey BJ. Nitrites. Encyclopedia of toxicology (second edition), 2005, 231-235.

4. Sun G. Prevention Medicine [In Chinese]. Beijing, People's Health Press, 2005, 164.

5. Jin P, Gangqiang D, Zhenhua G. Emergency response and prevention of food borne diseases [In Chinese]. Shanghai, Fudan University Press, 2006, 136-138.

6. Keqin Z et al. Investigation of an intoxication related to nitrite with unidentified label [In Chinese]. China Tropical Medicine, 2011, 11:1426-1427.

7. Fang $Y$, Liu $Q$, Zhang B. Investigation of food poison caused by cool food contaminated with sodium nitrite [In Chinese]. Chinese Journal of Public Health Management, 2012, 28:218.

8. Rongxian $R$, Haixiu AZ. Characteristic analysis of nitrite intoxication during 1994-2007 in Edan city, Hebei province, China [In Chinese]. Chinese Journal of Health Education, 2009, 25:718719.

9. YuHet al. Investigation on acute nitrite poisoning in Yangjiang city, Guangdong province, China [In Chinese]. Zhonghua Liu Xing Bing Xue Za Zhi, 2002, 23:419-421. pmid:12667350

10. Determination of nitrite and nitrate in foods-national food safety standard. (GB/T5009.33-2010) - National Standards of People's Republic of China [in Chinese]. Beijing, China Standards Press, 2010. 